\title{
Correction to: New Botryosphaeriales on native red milkwood (Mimusops caffra)
}

\author{
Fahimeh Jami $^{1} \cdot$ Seonju Marincowitz ${ }^{1} \cdot$ Bernard Slippers $^{1} \cdot$ Michael J. Wingfield ${ }^{1}$ \\ Published online: 4 July 2019 \\ (C) Australasian Plant Pathology Society Inc. 2019
}

\section{Correction to: Australasian Plant Pathology (2018) 47(5):475-484 https://doi.org/10.1007/s13313-018-0586-4}

Two new taxa, Neofusicoccum variabile and Pseudofusicoccum africanum were not validly published according to ICN (Shezhen) Art. 40.7.

The valid holotypifications are made and the new MycoBank identifiers are provided.

Neofusicoccum variabile Marinc., Jami \& M.J. Wingf. sp. nov. MB 831121.

Specimens examined: SOUTH AFRICA, Eastern Cape province, Haga Haga, Dec 2011, M. J. Wingfield, symptomatic twigs of Mimusops caffra (coastal red milkwood), holotype PREM 62174, ex-holotype CBS $143480=$ CMW 37739, paratype PREM 62176, living culture CBS 143482= CMW 37747. Additional specimens examined: PREM 62175, living culture CBS $143481=\mathrm{CMW} 37745$, other cultures CMW 37742, CMW 37748.

Pseudofusicoccum africanum Marinc., Jami \& M.J. Wingf. sp. nov. MB 831124.

Specimens examined: SOUTH AFRICA, Eastern Cape province, Haga Haga, Dec 2015, M. J. Wingfield, twigs of Mimusops caffra (coastal red milkwood), PREM 62172 holotype, ex-holotype CMW $48028=$ PPRI 25471. Additional specimens examined: PREM 62171, living culture CMW 48027, PREM 62173, living culture CMW 48030, other cultures CMW 48025, CMW 48026, CMW 48029, CMW 48035.

The online version of the original article can be found at https://doi.org/ 10.1007/s13313-018-0586-4

Fahimeh Jami

fahimeh.jami@fabi.up.ac.za

1 Department of Biochemistry, Genetics \& Microbiology, Forestry \& Agricultural Biotechnology Institute (FABI), University of Pretoria, Pretoria, South Africa 